Review

\title{
Age-related macular degeneration (AMD): More than meets the eye. The role of multimodal imaging in today's management of AMD
}

\author{
Robyn Guymer FRANZCO PhD ${ }^{1,2}$ and Zhichao Wu PhD ${ }^{1}$
}

1. Centre for Eye Research Australia, Royal Victorian Eye and Ear Hospital, Melbourne, Australia.

2. Ophthalmology, Department of Surgery, The University of Melbourne, Melbourne, Australia.

Correspondence: Prof Robyn Guymer, Centre for Eye Research Australia, Royal Victorian Eye and Ear Hospital, Melbourne, Australia Email: rhg@unimelb.edu.au

Short running title: Multimodal imaging in age-related macular degeneration management

Received 30 J une 2020; accepted 25 J uly 2020

Funding sources / Financial disclosure: NHMRC fellowship GNT1103013 to RHG Conflict of interest: RHG is on advisory boards of Novartis, Bayer, Roche/Genentech and Apellis, all work is outside the scope of this manuscript.

This is the author manuscript accepted for publication and has undergone full peer review but has not been through the copyediting, typesetting, pagination and proofreading process, which may lead to differences between this version and the Version of Record. Please cite this article as doi: 10.1111/ceo.13837

This article is protected by copyright. All rights reserved. 


\section{ABSTRACT}

Multimodal imaging (MMI) allows a more granular grading of age-related macular degeneration (AMD) disease severity, with many novel risk factors having been recently identified. With this imaging information, we are better able to counsel our patients with more accurate and individualized progression scenarios. MMI also allows identification of anatomical features that increase our understanding of disease processes involved in progression to late AMD. Treatment protocols for neovascular AMD (nAMD) depend largely on the optical coherence tomography (OCT) appearance to determine disease activity, which allows us to individualize treatment. In geographic atrophy (GA), new intervention trials require the ability to define the extent of GA, so that GA growth rate can be determined. This is achieved through fundus autofluorescence (FAF) imaging, which allows greater accuracy of border identification, as well as revealing FAF patterns predictive of growth rates. As we strive to bring interventions earlier in the disease course, OCT imaging provides an ability to identify the first signs of atrophy, which may serve as novel surrogate biomarkers for GA, thereby facilitating trials.

In the future, the use of artificial intelligence to automatically identify relevant features on MMI could further enhance our ability to determine disease severity, predict progression and assist in identifying disease activity parameters to support clinical decision making when treating nAMD. Newer developments may allow frequent, remote capturing of images, reducing clinic visits, detecting progression and monitoring neovascular activity in-between clinic visits. Being aware of these new imaging insights in AMD, greatly enhance our clinical management of AMD.

Keywords: Age-related macular degeneration (AMD), multi modal imaging, Clinical tests 


\section{INTRODUCTION}

\subsection{The humble colour fundus photograph and Beckman classification of}

AMD.

A dilated fundus examination with slit lamp biomicroscopy has been the mainstay for the diagnosis and review of individuals with AMD. Colour fundus photography (CFP) is the most common imaging modality in eyecare practices, and it provides a highresolution representation of the retinal appearance. CFP is useful for documenting the key AMD features seen on a clinical examination - drusen, pigmentary changes, geographic atrophy (GA) and neovascular complications. Grading AMD features on CFPs has formed the basis of severity scales for the past half century. However, over the past two decades, we have seen a dramatic improvement in our ability to image the retina. With it has come an enormous opportunity to discover new insights into the pathology of AMD, and to identify new markers of disease severity. Many of the new imaging techniques are making their way from research to the routine clinic, and this has led to an unprecedent opportunity to really understand where each individual stand in terms of disease severity and risk of progression. However, clinical examination and the CFP remain the most ubiquitous tool when seeing someone with AMD. As such, when the 2013 new classification of AMD was proposed - the Beckman classification - it was still based on CFP as the imaging modality to use to determine disease severity. ${ }^{1}$ The Beckman classification was developed using a consensus process undertaken by a group of AMD experts, because it was recognized at that time - and still to this date - that varied terminology is used when referring to AMD. The need to have a consistent terminology in AMD is more critical now, than ever before, as we start to have potential interventions at particular stages of the disease, and successful recruitment of the appropriate participants for these trial hinges on our ability to all talk about the disease in the same manner. 
Hence, it is a desire of many of those working in AMD that we all adopt the Beckman classification. (Table 1) The main features of the Beckman classification was to ensure that the term "dry AMD" was only used when an eye had late stage GA, and not to use the term - as is unfortunately often still the case - to refer to all AMD that is not neovascular. Thus, in Beckman, there are early and intermediate AMD stages, defined based on the size of drusen and the presence or absence of pigmentary changes attributable to AMD as seen on CFP. "Late AMD" is used to describe neovascular and atrophic AMD changes. The other major change in the Beckman classification, was to not label people with small drusen ( $<63 u m)$ as having AMD, but rather "normal ageing change", as their risk of vision loss from AMD was considered as not significantly above that of someone without any drusen. Thus, even in an era of MMI, CFP still remains immensely valuable for the initial grading of AMD severity.

\begin{tabular}{|l|l|}
\hline \multicolumn{2}{|l|}{ Table 1: Beckman Initiative for Macular Disease Classification System (2013)* } \\
\hline No ageing changes & No drusen or AMD pigmentary abnormalities^ \\
\hline $\begin{array}{l}\text { Normal ageing } \\
\text { changes }\end{array}$ & $\begin{array}{l}\text { Small drusen }(\leq 63 \mu \mathrm{m} \text { at their smallest diameter) and no } \\
\text { pigmentary abnormalities^ }\end{array}$ \\
\hline Early AMD & $\begin{array}{l}\text { Medium drusen }\left(>63 \mu \mathrm{m}^{\wedge} \text { and } \leq 125 \mu \mathrm{m}\right) \text { and no AMD } \\
\text { pigmentary abnormalities^ }\end{array}$ \\
\hline Intermediate AMD & $\begin{array}{l}\text { Large drusen (sized }>125 \mu \mathrm{m}) \text { and/or any AMD pigmentary } \\
\text { changes^ }\end{array}$ \\
\hline $\begin{array}{l}\text { Late AMD } \\
\begin{array}{l}\text { * Definition is based on the worst eye and for lesions assessed within two-disc } \\
\text { diameters of the fovea. } \\
\text { ^ AMD pigmentary abnormalities are hypo- or hyper-pigmentation associated } \\
\text { with presence of drusen }>63 \mu \mathrm{m} .\end{array}\end{array}$ \\
\hline
\end{tabular}

\subsection{Diagnosing late AMD}


Diagnosing the neovascular form of late AMD, whether or not obvious on clinical history, examination or CFP, has traditionally required invasive dye-based angiography, with fundus fluorescein angiography (FFA) (and more recently indocyanine green angiography [ICGA], for polypoidal choroidal vasculopathy) to confirm nAMD with the presence of abnormal leaking vessels. GA has always been defined on the basis of a CFP, but the extent of the atrophy is not always easy to determine on CFP alone. In the 1990s, fundus autofluorescence (FAF) imaging was developed that enabled imaging of the natural fluorescence of the retina emitted after stimulation by an excitation light source. Using a short-wavelength excitation source, imaged autofluorescence has been shown to primarily arise from the lipofuscin accumulated in the retinal pigment epithelium (RPE). ${ }^{2} \mathrm{FAF}$ imaging has proven valuable for assessment of AMD patients, particularly those with GA, as areas of atrophy appear hypo-autofluorescent due to the confluent loss of RPE cells. ${ }^{3-5}$ The boundaries of these atrophic areas are more clearly visualized on FAF imaging than on CFP (Fig. 1). As such, the FAF defined area of GA has now been accepted as an approved endpoint in clinical trials aiming to the slow growth of GA. There are particular patterns of autofluorescence in the areas surrounding the GA that can also be informative in predicting of the rate of disease progression. The presence of certain patterns hyper-autofluorescence surrounding areas of GA is often required as inclusion criteria for GA trials as this is considered to be associated with a more rapid GA growth rate. ${ }^{4,6}$

Figure 1: Geographic atrophy (GA) as seen on a colour fundus photograph (CFP; left) and fundus autofluorescence (FAF) imaging (right). The extent and borders of the multiple atrophic lesions are much easier seen in the FAF image compared to the CFP.

\section{MULTI-MODAL I MAGI NG: REVEALS MORE THAN MEETS THE EYE}




\subsection{Optical coherence tomography (OCT) in AMD - enhancing clinical assessments}

Since the first optical coherence tomography (OCT) images were published in 1993, it has quickly become one of the most useful imaging modalities in $\mathrm{AMD}^{7}$. It captures micrometre resolution images, utilising the different optical scattering properties of different cell types to provide a clearly delineated cross-sectional image of the retina. OCT can also provide en face visualisation of the retina and with enhanced depth imaging techniques it can also be utilised to allow the choroid to be better visualized. ${ }^{8}$ Most OCT machines today utilise spectral-domain technology (SD-OCT), which enables ultra-high resolution and fast image acquisition, whilst newer technology has led to swept-source OCT (SS-OCT). An international consensus group have published on terminology to be used when referring to particular zones on the OCT, with an additional consensus working group, the CAM recommending that OCT should be considered the base modality for assessing AMD.9,10

\subsection{An early marker of atrophy}

Modern high-resolution OCT, with its depth-resolved imaging, allows the visualisation of specific retinal layers affected by a disease process. In AMD, it has revealed earlier signs of the atrophic process, before lesions are clinically visible, nor detectable on CFPs or FAF. This technology offers the possibility of a classification system that is based on OCT-defined anatomical changes in the various layers of the retina, RPE and choroid as atrophy evolves. Photoreceptor cells, for example, contribute to multiple bands or zones in the OCT images: outer plexiform layer (OPL); synaptic terminals, Henle's fibre layer (HFL); axonal fibres, outer nuclear layer (ONL); cell bodies, external limiting membrane (ELM); junctions with Müller glia, ellipsoid zone (EZ); ellipsoid part of photoreceptor inner segments, outer segments (OS), and interdigitation zone (IZ); interface of outer segments with RPE 
apical processes. Clinically, we infer photoreceptor health based upon the integrity of these bands or zones.

Following a longitudinal natural history cohort of cases with large drusen in at least one eye and performing MMl every 3 months, our group was able to recognize OCT features, that precede the development of atrophy and we termed these features nascent geographic atrophy (nGA). ${ }^{11}$ The features include: (i) the subsidence of the inner nuclear layer (INL) and outer plexiform layer (OPL) and/or (ii) the presence of a hyporeflective wedge-shaped band within the Henle's fiber layer portion of the OPL, both indicating a loss of the photoreceptor layer. (Fig. 2) Whilst we observed that the presence of one or both of these signs uniquely precede the development of atrophy, these changes are often seen associated with ELM, EZ or IZ loss. In addition, it is usual that underlying the signs signifying photoreceptor loss, there is often an attenuation or disruption of the RPE and a hyper-transmission of the signal into the choroid. These additional OCT signs add to the phenotypic profile of people who would still be classified as either early or intermediate AMD according to the Beckman classification, yet they have clear signs that cell death has already occurred, and the atrophic process is underway.

Figure 2: Nascent geographic atrophy (nGA); colour fundus photography of an eye with large drusen and hyper pigmentary changes (left) and an optical coherence tomography B-scan (right) shows the first signs of cell loss and the onset of atrophy, with (i) subsidence of the inner nuclear layer (INL) and outer plexiform layer (OPL; vertical arrow), and (ii) hyporeflective wedge-shaped band (diagonal arrows) present.

We recently determined that amongst individuals with bilateral large drusen that developed nGA, there was a 78-fold increased rate of progression to GA as 
compared to eyes that did not develop nGA when followed over 3 years. ${ }^{12}$ This was a similar finding to a previous study that demonstrated that eyes with nGA at baseline had a 56-fold increased risk of developing GA over an average follow-up period of 9 years. ${ }^{13}$

Currently, there are no specific treatments (apart from lifestyle choices and supplements) proven to prevent or delay the progression to late stages of AMD, which are urgently needed as our populations age. However, the slowly progressive nature of AMD means that the evaluation of potential interventions for preventing or delaying the progression of AMD from its early stages to late complications remains a challenge. This necessitates trials with large cohorts followed over long periods to detect a meaningful treatment effect. This is a significant barrier for evaluating new promising interventions for early stages of AMD. In 2017 however, the United States Food and Drug Administration (FDA) suggested that preventing photoreceptor loss would be considered a clinically meaningful trial endpoint given the established link between photoreceptor loss and visual function. ${ }^{14}$ Since evidence suggests that nGA is a strong predictor for the development of GA, there is potential for nGA to be a useful surrogate endpoint in preventative treatment trials. It was used as a combined atrophic endpoint with GA in the recent Laser Intervention in the Early Stages of AMD (LEAD) randomised-controlled trial exploring subthreshold nanosecond laser as potential intervention to slow AMD progression. ${ }^{15}$ We are now working to establish if nGA can fulfil criteria required of a surrogate endpoint, as if an acceptable endpoint by regulatory authorities, it could be used to assess efficacy of novel interventions that aim to slow progression of the early stages of AMD.

To further address the issue of AMD classification in the era of MMI, an international group of AMD and retinal imaging experts assembled for a series of meetings called the Classification of Atrophy Meetings (CAM). This group met to review existing data 
and propose a consensus definition and nomenclature for OCT-defined atrophy in the setting of AMD. At CAM, it was agreed that whilst there was a wide availability of multiple imaging methods to evaluate the fundus and atrophy, it was necessary to choose a base method of imaging, where other methods would be confirmatory. The CAM consensus recommendation was that OCT should serve as the reference method for defining different atrophy phenotypes and stages. However, the CAM group recognized that OCT alone may not be sufficient in many cases, and that other imaging methods such as FAF may provide additional information. From CAM came a series of OCT-defined terms based on the layers of the outer retina that are affected as cell death, related to the photoreceptors and RPE, occurs in atrophy. Complete RPE and outer retinal atrophy (CRORA) was proposed as a definition for atrophy that occurred in association with drusen, and it was defined by the following 4 criteria: (1) a region of hypertransmission of $\geq 250 \mu \mathrm{m}$ in diameter, and (2) a zone of attenuation or disruption of the RPE of $\geq 250 \mu \mathrm{m}$ in diameter, and (3) evidence of overlying photoreceptor degeneration, all occurring in the absence of signs of an RPE tear. ${ }^{16}$ The term incomplete RPE and outer retinal atrophy (iRORA) was introduced to describe a stage of early cell loss in AMD where these OCT signs were present, but they did not fulfil all the criteria for CRORA. ${ }^{16,17}$ (Table 2) (Fig. 3)

\begin{tabular}{|l|l|}
\hline Table 2 cRORA and iRORA \\
\hline Term & OCT findings \\
\hline Complete RPE and & $\bullet$ Zone of hypertransmission of $\geq 250 \mu \mathrm{m}$ \\
outer retinal atrophy & $\bullet$ Zone of attenuation or disruption of the RPE \\
(cRORA) & band of $\geq 250 \mu \mathrm{m}$ \\
& Evidence of overlying photoreceptor \\
& degeneration whose features include outer \\
& nuclear layer thinning, external limiting \\
& membrane loss and ellipsoid zone loss. \\
& Exclude: signs of RPE tear \\
\hline
\end{tabular}




\begin{tabular}{|c|c|}
\hline $\begin{array}{l}\text { Incomplete RPE and } \\
\text { outer retinal atrophy } \\
\text { (iRORA) }\end{array}$ & 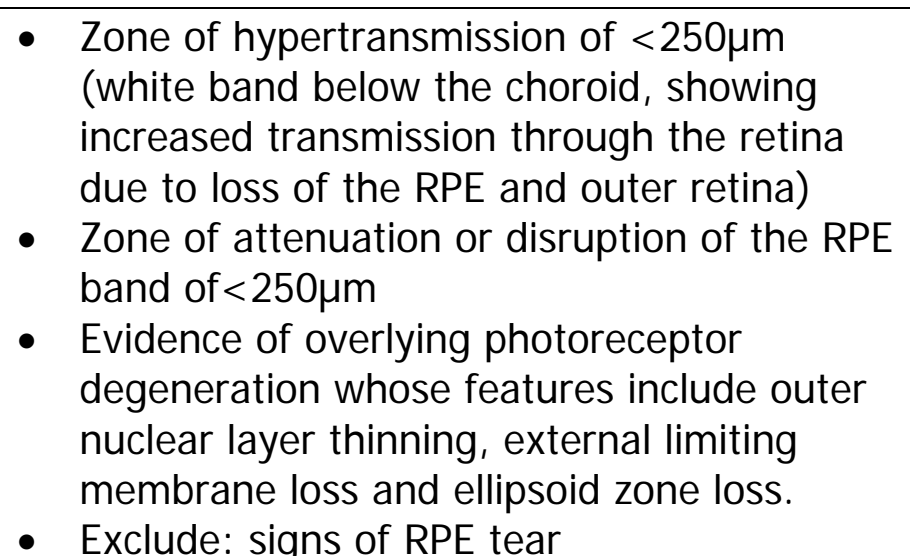 \\
\hline
\end{tabular}

Figure 3: Complete retinal pigment epithelium (RPE) and outer retinal atrophy (CRORA; left) and incomplete RPE and outer retinal atrophy (iRORA; right). The optical coherence tomography (OCT) B-scan for the CRORA (left) includes the following features: (i) a region of hypertransmission of $\geq 250 \mu \mathrm{m}$ in diameter (white line), (ii) a zone of attenuation or disruption of the RPE of $\geq 250 \mu \mathrm{m}$ in diameter (between long arrows) and (iii) evidence of overlying photoreceptor degeneration with loss of outer retinal layers, including the external limiting membrane (ELM) and ellipsoid zone (EZ) disruption (between short arrows). The OCT B-scan for the iRORA (right) includes the following features that describes a stage of early cell loss that did not fulfil all the criteria for CRORA: (i) a region of hypertransmission of $<250 \mu \mathrm{m}$ in diameter (white line), (ii) a zone of disruption of the RPE (between long arrows) and (iii) evidence of overlying photoreceptor degeneration with thinning of the outer nuclear layer (ONL) and loss of the ELM and EZ disruption (between short arrows). In this example there is no subsidence of the inner nuclear layer (INL) and outer plexiform layer (OPL), nor is there evidence of any hyporeflective wedge-shaped bands within the Henle's fiber layer portion of the OPL. As such, the criteria for nascent geographic atrophy (nGA) as originally described, are not met. 
The CAM group felt that the well-entrenched term of geographic atrophy (GA) should continue to be used as a term applied to the subset of CRORA occurring in the absence of macular neovascularization (MNV). Similarly, CAM suggested that the term nGA be used more broadly when OCT defined iRORA was present in the absence of MNV.

Whilst nGA tends to be used as a clinical term, which is considered interchangeable with iRORA, its original definition and one that has an established risk of progression GA requires subsidence of the INL and OPL and/or the presence of a hyporeflective wedge-shaped band within the Henle's fiber layer portion of the OPL to be present as signs of photoreceptor loss. nGA does not require RPE changes and hypertransmission through to the choroid, which is required in the definition of iRORA. It remains to be seen what the importance of change in each band or zone will ultimately be for predicting progression to vision loss. Longitudinal studies incorporating detailed grading will provide the necessary data to determine the final definition of a robust, reproducibly graded and predictive set of signs of AMD progression. Studies to advance this understanding are currently under intense research. The reproducibility for identifying iRORA is crucial, and it will undoubtedly improve as clinicians and reading centers become more familiar with the OCT findings, as well as with advances in OCT technology and adoption of artificial intelligence (Al) algorithms as they become available.

\subsection{OCT identified anatomical risk factors in AMD}

There are well-described, characteristic fundus features that are associated with a higher risk of AMD for progression. The Age-Related Eye Disease Study (AREDS) described the risk of disease progression conferred by different sized drusen and pigmentary changes within the central macula as identified on CFP. ${ }^{18}$ More recently, multimodal imaging (MMI), including OCT, has identified other features that appear 
to increase the risk of vision loss. These anatomical changes include intraretinal hyperreflective foci, heterogeneous internal reflectivity within drusen, reticular pseudodrusen (RPD), also known as subretinal drusenoid deposits (SDD), and a shallow irregular RPE elevation (SIRE). These are considered as high-risk OCT biomarkers for progression, many of which can also be confirmed on other MMI modalities such as FAF and near-infrared reflectance (NIR) imaging.

\subsubsection{I ntraretinal Hyperreflective Foci}

On OCT, intraretinal retinal hyperreflective foci are characterized by discrete lesions, in a single or clustered distribution, that are of equal or greater reflectivity than that of the RPE. Often, they are observed overlying drusen, where they are thought to represent dissociated RPE cells that have migrated towards the inner retina. In AMD, macular hyperpigmentation seen on CFP has high spatial correlation to OCT hyperreflective foci and often represents the same anatomical lesion. ${ }^{19}$ (Fig. 4) Hyperreflective foci are associated with an increased risk of progression from early to late AMD, where greater hyperreflective foci quantity and their inner retinal migration are considered to increase the risk of progression to GA. 2021

Figure 4: Hyperreflective foci (HRF). Colour fundus photography of an eye with large drusen and hyperpigmentary changes (left), and an optical coherence tomography (OCT) B-scan taken through an area with hyperpigmentary changes (shown on the right) reveals two hyperreflective foci (white arrows) sitting above the apex of large drusen. Note the choroidal signal hypotransmission (or shadow) below the HRF.

\subsubsection{Heterogeneity of Drusen}

Drusen, seen clinically as pale, yellow deposits, are the hallmark of AMD. Their clinical characteristics, as defined on CFP, are a main contributor to determining 
risk. $^{22}$ On OCT, drusen are seen as accumulation of material between the RPE and Bruch's membrane. However, they can have varied appearance as revealed by OCT imaging, with a variety of shapes and ultrastructural features which have been reported to influence the risk profile. ${ }^{23-25}$ As such, it has been proposed that future grading systems should also take into consideration drusen morphological considerations such as shape, reflectivity, and homogeneity when considering disease severity and risk of progression. ${ }^{23}$

On OCT drusen can appear with what has been termed as, heterogeneous internal reflectivity within the drusen (HIRD), which histologically have been found to have deposits of hydroxyapatite, a form of calcium apatite. Intense lines of hyperreflectivity in or near Bruch's membrane (fourth reflective band) - referred to as hyperreflective crystalline deposits (HCDs), that correlate with cholesterol crystals - can also be seen and correspond with refractile lesions on CFP. ${ }^{26}$ Both deposits have also been strongly associated with progression to late AMD, particularly GA. ${ }^{24-26}$

Figure 5: Heterogenous internal reflectivity within drusen seen on an optical coherence tomography (OCT) B-scan (right; long arrows), associated with an intense line of hyper-reflectivity near Bruch's membrane (short arrow; termed hyperreflective crystalline deposits [HCDs]), seen in an eye with large drusen, pigmentary abnormalities and refractile lesions on colour fundus photography (left).

\subsubsection{Reticular pseudodrusen (RPD)}

High-resolution MMl has revealed a further distinct drusen phenotype - reticular pseudodrusen (RPD) or subretinal drusenoid deposits (SDD). ${ }^{27,28}$ Whilst RPD can be recognized on clinical fundus examination or on CFP, over $50 \%$ are not seen on CFP. ${ }^{29-31}$ When seen on CFP, they appear as either distinct small white dots, often confused with small hard drusen, or as faint yellowish interlacing networks (Fig 6). They do not form part of the Beckman classification of AMD severity. MMI has 
revealed that RPD are much more common than initially thought, where they are often best seen on OCT, as deposits located internal to the RPE, in contrast to traditional drusen which are located external to the RPE. (Fig. 6) ${ }^{27,28}$ On OCT, they can appear as diffuse accumulations of material on the RPE extending to the EZ (Stage 1), or as shallow mound-like accumulations of material on the RPE elevating the EZ (Stage 2). They can also form conical (haystack-like) projections from the RPE extending across the EZ (Stage 3). ${ }^{32}$ This material appears as hyporeflective lesions on IR imaging, whilst on FAF they can appear as hypo-autofluorescent lesions sometimes with a hyper-autofluorescent center. ${ }^{33}$

Figure 6: Reticular pseudodrusen (RPD). (A) Colour fundus photograph (CFP) showing RPD as both small pale dots and a broader, faint net-like pattern (superiorly); region outlined by the dashed rectangle is shown as a magnified insert in [C]); (B) near-infrared reflectance (NIR) imaging showing RPD and target-like lesions (region outlined by the dashed rectangle is shown as a magnified insert in [D]); (E) optical coherence tomography (OCT) B-scan shows RPD as peaks (haystack appearance; short arrows) above the retinal pigment epithelium (RPE); and through the ellipsoid zone, and conventional drusen (example in long arrow) below the RPE. (region outlined by the dashed box is shown as a magnified insert in $[\mathbf{F}])$

The interest in RPD has increased exponentially due to a new appreciation from several longitudinal cohort studies, with appropriate MMI, that RPD are a strong predictor for progression to late AMD. ${ }^{34-36}$ Due to their correlation with progression, it could be argued that an MMI approach is mandatory in the management of AMD so that, at the very least, RPD can be identified and patients counselled accordingly. Because these deposits have only been reliably identified using modern imaging 
techniques, their aetiology remain unknown and is under intense investigation. ${ }^{30}$ 37,38

\subsubsection{Shallow irregular RPE elevation (SIRE) and OCT angiography (OCTA)}

OCT has also provided insight into potential anatomical features that specifically increase the risk of nAMD. This is particularly important as it offers an opportunity to monitor high-risk patients more closely and for clinicians to stress the importance of regular home monitoring to this subgroup, as it is clear that early detection and timely intervention with highly effective anti-VEGF treatments is essential for best outcomes. 39,40

Asymptomatic, sub-RPE, type 1 non-exudative macular neovascularization (NE-MNV) in eyes with drusen have been identified on dye-based angiography. With the emergence of OCT angiography (OCTA), they can be identified without invasive angiography. ${ }^{41}$ Like RPD, these lesions, which increase the risk of exudative nAMD, are being identified more often in routine imaging of asymptomatic early stage AMD cases than had otherwise been considered the case. ${ }^{42,43}$ Indeed, whilst they do appear to represent eyes at greater risk of nAMD, they may also protect against hypoxia and cell death by recapitulating the choroidal vessels. ${ }^{44,45}$

To date, although increasingly available, OCTA is currently largely confined to retinal subspeciality clinics, where it is often used to visualize neovascular networks in nAMD (Fig. 7), or in clinical research groups. OCTA is not yet readily available in daily clinical practice.

Figure 7: (A) A newly diagnosed neovascular complex seen on the swept-source optical coherence tomography angiography (SS-OCTA) en face flow image, generated from a slab (B) between the outer retina to choriocapillaris (ORCC) slab 
(from the junction between the outer plexiform layer and outer nuclear layer to $8 \mu \mathrm{m}$ below Bruch's membrane) on the SS-OCTA B-scan (dashed yellow lines) taken through the area indicated by the white arrow in $[\mathbf{A}]$. The vessel complex on the en face image is visible as hyperreflective material on the B-scan, and it is associated with subretinal fluid. (C) SS-OCTA en face flow image and (D) B-scan taken through the region indicated by the white arrow, one month after the first treatment with anti-vascular endothelial growth factor, showing that the abnormal vessel complex is no longer visible.

However, there are signs, visible on SD-OCT, that when present, would heighten suspicion that these abnormal vessel complexes may be present. Since these type 1 neovascular lesions reside under the RPE and above Bruch's membrane, they produce what has been described as the "double-layer sign". This sign was initially described in eyes with polypoidal choroidal vasculopathy, ${ }^{46}$ consisting of two highly reflective layers - the RPE, and another beneath the RPE, with moderately reflective material between the two. ${ }^{46,47}$

We recently described key common structural characteristics of the RPE elevations seen on OCT that were suggestive for the presence of NE-MNV. ${ }^{48}$ We described a shallow, irregular RPE elevation (SIRE) above Bruch's membrane, with an arbitrary length $\geq 1000 \mu \mathrm{m}$ of shallow elevation and a height above Bruch's membrane of predominantly $<100 \mu \mathrm{m}$. (Fig. 8) We identified these features in $10 \%$ of eyes with large drusen and when identified, it had a positive predictive value of $25 \%$; where 1 in 4 cases with SIRE will have NE-MNV on OCTA, and the negative predictive value of $100 \%$ for NE-MNV. We suggest the SIRE sign can be used to screen for NE-MNV, which significantly increases the risk of nAMD. ${ }^{48}$

Figure 8: (A) An example of an eye with large drusen and pigmentary changes seen on colour fundus photography. (B) A spectral-domain optical coherence 
tomography (OCT) B-scan, taken through the region indicated by the horizontal white arrow on [A] shows a region of shallow irregular retinal pigment epithelium (RPE) elevation (SIRE) above Bruch's membrane (white line). Presence of a SIRE sign increases suspicion for the presence of non-exudative macular neovascularisation (NE-MNV), which can be detected on OCT angiography (OCTA). Note that a large druse with hyperreflective foci is also present nasal to the fovea on the OCT B-scan. (C) A different example where the SIRE sign (indicated by the region between the two vertical white arrows) was detected on a swept-source OCTA B-scan. (D) An en face flow image, generated from a slab between the outer retina to choriocapillaris (ORCC) slab (from the junction between the outer plexiform layer and outer nuclear layer to $8 \mu \mathrm{m}$ below Bruch's membrane; yellow dashed lines in [C]) revealed the presence of an NE-MNV.

OCTA imaging of the microvasculature of the retina and choroid will continue to be developed and will make its way into clinical care. An increased understanding of changes in choroidal blood flow is currently very topical and integral to understanding the progression and the development of nAMD and GA.

\subsubsection{OCT imaging blurs the line between "dry" and "wet" AMD. I mportant implications for the treatment of nAMD}

Imaging with OCT and OCTA have provided insights that are blurring the lines between "dry" and "wet" AMD. Whilst OCTA detects NE-MNV in eyes with the early stages of AMD ("dry", but potentially "wet"), OCT detects subretinal fluid (SRF) in eyes that do not appear to have any evidence of abnormal choroidal vessels ("wet", but actually "dry"). 49,50 We have previously referred to the latter as non-exudative detachment of the neurosensory retina (NEDNR) and suggest that there are a variety of reasons that SRF can exist without active neovascular disease. (Fig. 9) 
Figure 9: An optical coherence tomography (OCT) B-scan (right) in an eye with large drusen and hyperpigmentary abnormalities on colour fundus photography (left) reveals subretinal fluid under the fovea (arrow). This eye did not have evidence of neovascular complications and thus has non-exudative detachment of the neurosensory retina (NEDNR).

These are important findings as we consider the end goal of treatments for nAMD. As individualizing treatments, based upon disease activity, are now standard in antivascular endothelial growth factor (VEGF) treatment protocols for nAMD, such as in treat-and-extend (T\&E), the definition of active disease is important. Current protocols looking to individualize treatment for nAMD aim to completely "dry" the retina (i.e. continue treatment until there are no signs of retinal fluid), assuming that returning the retinal anatomy to as near normal as possible is the desired goal. It is assumed that resolution of all fluid - intraretinal, subretinal and even sub-retinal pigment epithelium (RPE) fluid - will lead to better visual acuity (VA) outcomes. In a T\&E protocol, this would dictate monthly treatment intervals until the retina was "dry", with interval extensions not possible until a "dry" retina had been achieved. However, there are now several studies showing that there is not a positive correlation between a "dry" retina and VA outcomes. ${ }^{51,52}$ The FLUID study was also able to show in a randomized controlled trial that non-inferior VA results could be achieved with tolerating some SRF whilst using a T\& E protocol. ${ }^{53}$ More recent studies have suggested that some SRF should indeed be tolerated, as it resulted in better visual outcomes and affords some protection from the development of atrophy. ${ }^{54}$ Persistent of SRF, whilst undergoing a course of anti-VEGF treatment, may indicate that there is a still a vascular network present. This network is capable of delivering oxygen to viable but dysfunctional RPE and photoreceptors, offering some protection from cell death and subsequent macular atrophy. These studies call into question what the ultimate aim of treatment should be and confirms that 
regular high-quality OCT imaging and up to date knowledge of new clinical insights on OCT interpretation is essential to guide treatment decisions, to ensure best longterm VA outcomes with the minimal number of treatments.

\section{CONCLUSI ON: THE I MAGI NG FUTURE}

The rapid advances in imaging technology has led to an enormous increase in our ability to understand disease processes occurring in AMD. Using MMI in a clinical setting improves our ability to accurately stage the disease and determine which anatomical risk factors are present. This should influence our counselling of each patient and our management strategy we put in place for each case. It is clear that subgrouping cases, based upon imaging criteria, will likely lead to more targeted clinical trials with different interventions for different subgroups. However, with the ageing population and expected increase in the number of people seeking management of their AMD, we will not be able to review as we currently do. Yet being able to image the state of the retina at regular intervals is integral to providing the care required. Recognizing that remote monitoring (potentially in the home) is a necessary new way of delivering care has led to novel inventions to help deliver the tools that will be required. These tools are important not only for monitoring early disease progression, but also in the management of nAMD treatments to reduce clinic visits, as longer duration treatments become available (e.g. a port delivery system). ${ }^{55}$ Home monitoring through a portable, relatively inexpensive, and selfoperated OCT would allow almost continuous monitoring, to truly individualize treatment regimens and requirements in order to optimize outcomes. ${ }^{56}$ Such imaging can be combined with artificial intelligence (Al) enabled software to analyse the data automatically and communicate directly with the patient regarding actions required. This type of approach may well transform the way retinal diseases such as AMD are managed in the future. 
OCT images offer millions of individual data points, providing extensive information about retinal morphology, much of which may not be visible to the naked eye or interpretable by the clinician. Interpreting this rich imaging data in retinal disease has become vastly more complex, with individual patients providing their own "big data." Intelligent tools are required to ensure we make the most of our imaging capabilities in retinal diseases. Al tools are being developed that will be used to identify, localize and quantify pathological features and to develop robust predictive models of disease progression. These tools will dramatically improve diagnostic accuracy and our ability to screen, establish prognosis, and provide support for clinical decision making. It will also help detect disease recurrences and to identify targets for new interventions. ${ }^{57}$ Retinal multimodal imaging and Al methodologies should be exploited to provide a personalized medicine approach, as our health care systems will no longer be able to provide ongoing face-to-face routine reviews for everyone that appears at risk of a vision threatening complication of common chronic diseases of the retina such as AMD. 


\section{REFERENCES}

1. Ferris, F.L., et al., Clinical Classification of Age-related Macular Degeneration. Ophthalmology, 2013. 120(4): p. 844-851.

2. Weiter, J.J., et al., Retinal-Pigment Epithelial Lipofuscin and Melanin and Choroidal Melanin in Human Eyes. Investigative Ophthalmology \& Visual Science, 1986. 27(2): p. 145-152.

3. Spaide, R.F., Fundus autofluorescence and age-related macular degeneration. Ophthalmology, 2003. 110(2): p. 392-399.

4. Schmitz-Valckenberg, S., et al., Fundus Autofluorescence and Progression of Age-related Macular Degeneration. Survey of Ophthalmology, 2009. 54(1): p. 96-117.

5. Bearelly, S. and S.W. Cousins, Fundus Autofluorescence Imaging in AgeRelated Macular Degeneration and Geographic Atrophy. Retinal Degenerative Diseases: Laboratory and Therapeutic Investigations, 2010. 664: p. 395-402.

6. Holz, F.G., et al., Progression of geographic atrophy and impact of fundus autofluorescence patterns in age related macular degeneration. American Journal of Ophthalmology, 2007. 143(3): p. 463-472.

7. Fercher, A.F., et al., In-Vivo Optical Coherence Tomography. American J ournal of Ophthalmology, 1993. 116(1): p. 113-115.

8. Drexler, W., et al., Ultrahigh-resolution ophthalmic optical coherence tomography. Nature Medicine, 2001. 7(4): p. 502-507.

9. Staurenghi, G., et al., Proposed lexicon for anatomic landmarks in normal posterior segment spectral-domain optical coherence tomography: the IN*OCT consensus. Ophthalmology, 2014. 121(8): p. 1572-8.

10. Holz, F.G., et al., Imaging Protocols in Clinical Studies in Advanced Age-Related Macular Degeneration: Recommendations from Classification of Atrophy Consensus Meetings. Ophthalmology, 2017. 124(4): p. 464-478. 
11. Wu, Z, et al., Optical coherence tomography-defined changes preceding the development of drusen-associated atrophy in age related macular degeneration. Ophthalmology, 2014. 121(12): p. 2415-22.

12. Wu, Z, et al., Prospective Longitudinal Evaluation of Nascent Geographic Atrophy in Age-Related Macular Degeneration. Ophthalmol Retina, 2020. 4(6): p. 568-575.

13. Ferrara, D., et al., Optical Coherence Tomography Features Preceding the Onset of Advanced Age-Related Macular Degeneration. Investigative Ophthalmology \& Visual Science, 2017. 58(9): p. 3519-3529.

14. Csaky, K., et al., Report From the NEI/FDA Endpoints Workshop on AgeRelated Macular Degeneration and Inherited Retinal Diseases. Investigative Ophthalmology \& Visual Science, 2017. 58(9): p. 3456-3463.

15. Guymer, R.H., et al., Subthreshold Nanosecond Laser Intervention in AgeRelated Macular Degeneration The LEAD Randomized Controlled Clinical Trial. Ophthalmology, 2019. 126(6): p. 829-838.

16. Sadda, S.R., et al., Consensus Definition for Atrophy Associated with AgeRelated Macular Degeneration on OCT Classification of Atrophy Report 3. Ophthalmology, 2018. 125(4): p. 537-548.

17. Guymer, R.H., et al., Incomplete Retinal Pigment Epithelial and Outer Retinal Atrophy in Age-Related Macular Degeneration Classification of Atrophy Meeting Report 4. Ophthalmology, 2020. 127(3): p. 394-409.

18. Age-related Eye Disease Study Reserch Group, The age-related eye disease study system for classifying age related macular degeneration from stereoscopic color fundus photographs: The Age-Related Eye Disease Study Report Number 6. American Journal of Ophthalmology, 2001. 132(5): p. 668681.

19. Folgar, F.A., et al., Spatial Correlation between Hyperpigmentary Changes on Color Fundus Photography and Hyperreflective Foci on SDOCT in Intermediate 
$A M D$. Investigative Ophthalmology \& Visual Science, 2012. 53(8): p. 46264633.

20. Christenbury, J.G., et al., Progression of Intermediate Age-related Macular Degeneration with Proliferation and Inner Retinal Migration of Hyperreflective Foci. Ophthalmology, 2013. 120(5): p. 1038-1045.

21. Nassisi, M., et al., Quantity of Intraretinal Hyperreflective Foci in Patients With Intermediate Age-Related Macular Degeneration Correlates With 1-Year Progression. Investigative Ophthalmology \& Visual Science, 2018. 59(8): p. 3431-3439.

22. Ferris, F.L., et al., A simplified severity scale for age-related macular degeneration - AREDS report no. 18. Archives of Ophthalmology, 2005. 123(11): p. 1570-1574.

23. Khanifar, A.A., et al., Drusen Ultrastructure Imaging with Spectral Domain Optical Coherence Tomography in Age-related Macular Degeneration. Ophthalmology, 2008. 115(11): p. 1883-1890.

24. Tan, A.C.S., et al., Calcified nodules in retinal drusen are associated with disease progression in age-related macular degeneration. Science Translational Medicine, 2018. 10(466).

25. Oishi, A., et al., Prevalence, Natural Course, and Prognostic Role of Refractile Drusen in Age-Related Macular Degeneration. Investigative Ophthalmology \& Visual Science, 2017. 58(4): p. 2198-2206.

26. Fragiotta, S., et al., The Fate and Prognostic Implications of Hyperreflective Crystalline Deposits in Nonneovascular Age-Related Macular Degeneration. Investigative Ophthalmology \& Visual Science, 2019. 60(8): p. 3100-3109.

27. Greferath, U., et al., Correlation of Histologic Features with In Vivo Imaging of Reticular Pseudodrusen. Ophthalmology, 2016. 123(6): p. 1320-31.

28. Zweifel, S.A., et al., Reticular Pseudodrusen Are Subretinal Drusenoid Deposits. Ophthalmology, 2010. 117(2): p. 303-U139. 
29. Arnold, J. . ., et al., Reticular Pseudodrusen - a Risk Factor in Age-Related Maculopathy. Retina-the J ournal of Retinal and Vitreous Diseases, 1995. 15(3): p. 183-191.

30. Wu, Z.C., et al., Reticular Pseudodrusen in Intermediate Age-Related Macular Degeneration: Prevalence, Detection, Clinical, Environmental, and Genetic Associations. Investigative Ophthalmology \& Visual Science, 2016. 57(3): p. 1310-1316.

31. Finger, R.P., et al., Reticular Pseudodrusen and Their Association with AgeRelated Macular Degeneration: The Melbourne Collaborative Cohort Study. Ophthalmology, 2016. 123(3): p. 599-608.

32. Suzuki, M., T. Sato, and R.F. Spaide, Pseudodrusen subtypes as delineated by multimodal imaging of the fundus. Am J Ophthalmol, 2014. 157(5): p. 100512.

33. Ueda-Arakawa, N., et al., Sensitivity and specificity of detecting reticular pseudodrusen in multimodal imaging in Japanese patients. Retina, 2013. 33(3): p. 490-7.

34. Finger, R.P., et al., Reticular pseudodrusen: a risk factor for geographic atrophy in fellow eyes of individuals with unilateral choroidal neovascularization. Ophthalmology, 2014. 121(6): p. 1252-6.

35. Schmitz-Valckenberg, S., et al., Reticular Drusen Associated with Geographic Atrophy in Age-Related Macular Degeneration. Investigative Ophthalmology \& Visual Science, 2011. 52(9): p. 5009-5015.

36. Gil, J.Q., et al., Clinical features and long-term progression of reticular pseudodrusen in age-related macular degeneration: findings from a multicenter cohort. Eye, 2017. 31(3): p. 364-371.

37. Spaide, R.F., S. Ooto, and C.A. Curcio, Subretinal drusenoid deposits AKA pseudodrusen. Survey of Ophthalmology, 2018. 63(6): p. 782-815. 
38. Wightman, A.J. and R.H. Guymer, Reticular pseudodrusen: current understanding. Clinical and Experimental Optometry, 2019. 102(5): p. 455462.

39. Lim, J.H., et al., Delay to Treatment and Visual Outcomes in Patients Treated With Anti-Vascular Endothelial Growth Factor for Age-Related Macular Degeneration. American J ournal of Ophthalmology, 2012. 153(4): p. 678-686.

40. Finger, R.P., et al., Predictors of anti-VEGF treatment response in neovascular age-related macular degeneration. Survey of Ophthalmology, 2014. 59(1): p. 1-18.

41. Lane, M., et al., Visualizing the Choriocapillaris Under Drusen: Comparing 1050nm Swept-Source Versus 840-nm Spectral-Domain Optical Coherence Tomography Angiography. Investigative Ophthalmology \& Visual Science, 2016. 57(9): p. Oct585-Oct590.

42. Dias, J.R.D., et al., Natural History of Subclinical Neovascularization in Nonexudative Age-Related Macular Degeneration Using Swept-Source OCT Angiography. Ophthalmology, 2018. 125(2): p. 255-266.

43. Roisman, L., et al., Optical Coherence Tomography Angiography of Asymptomatic Neovascularization in Intermediate Age-Related Macular Degeneration. Ophthalmology, 2016. 123(6): p. 1309-1319.

44. Shi, Y., et al., Predictive Value of the OCT Double-Layer Sign for Identifying Subclinical Neovascularization in Age-Related Macular Degeneration. Ophthalmol Retina, 2019. 3(3): p. 211-219.

45. Pfau, M., et al., Type 1 Choroidal Neovascularization Is Associated with Reduced Localized Progression of Atrophy in Age-Related Macular Degeneration. Ophthalmol Retina, 2020. 4(3): p. 238-248.

46. Sato, T., et al., Tomographic features of branching vascular networks in polypoidal choroidal vasculopathy. Retina-the J ournal of Retinal and Vitreous Diseases, 2007. 27(5): p. 589-594. 
47. Querques, G., et al., Functional Characterization and Multimodal Imaging of Treatment-Naive "Quiescent" Choroidal Neovascularization. Investigative Ophthalmology \& Visual Science, 2013. 54(10): p. 6886-6892.

48. Narita, C., et al., Structural OCT Signs Suggestive of Subclinical Nonexudative Macular Neovascularization in Eyes with Large Drusen. Ophthalmology, 2020. 127(5): p. 637-647.

49. Guymer, R.H., Age-Related Macular Degeneration: Not So Wet and Dry. Ophthalmol Retina, 2020. 4(3): p. 249.

50. Lek, J.J., et al., Interpretation of Subretinal Fluid Using OCT in Intermediate Age-Related Macular Degeneration. Ophthalmol Retina, 2018. 2(8): p. 792802.

51. Heier, J.S., et al., Intravitreal Aflibercept (VEGF Trap-Eye) in Wet Age related Macular Degeneration. Ophthalmology, 2012. 119(12): p. 2537-2548.

52. Martin, D.F., et al., Ranibizumab and Bevacizumab for Treatment of Neovascular Age-related Macular Degeneration: Two-Year Results. Ophthalmology, 2012. 119(7): p. 1388-1398.

53. Guymer, R.H., et al., Tolerating Subretinal Fluid in Neovascular Age-Related Macular Degeneration Treated with Ranibizumab Using a Treat-and-Extend Regimen FLUID Study 24-Month Results. Ophthalmology, 2019. 126(5): p. 723-734.

54. Zarbin, M.A., I. Stoilov, and L. Hill, Anti-VEGF Resistant Subretinal Fluid is Associated With Reduced Risk of Macular Atrophy and Better Visual Acuity: Drug-Induced Choroidal New Vessel Homeostasis? Investigative Ophthalmology \& Visual Science, 2020. 61(7): p. 3503-3503.

55. Campochiaro, P.A., et al., The Port Delivery System with Ranibizumab for Neovascular Age-Related Macular Degeneration: Results from the Randomized Phase 2 Ladder Clinical Trial. Ophthalmology, 2019. 126(8): p. 1141-1154. 
56. Maloca, P., et al., Safety and Feasibility of a Novel Sparse Optical Coherence Tomography Device for Patient-Delivered Retina Home Monitoring. Translational Vision Science \& Technology, 2018. 7(4).

57. Schmidt-Erfurth, U., et al., Machine Learning to Analyze the Prognostic Value of Current Imaging Biomarkers in Neovascular Age-Related Macular Degeneration. Ophthalmol Retina, 2018. 2(1): p. 24-30. 


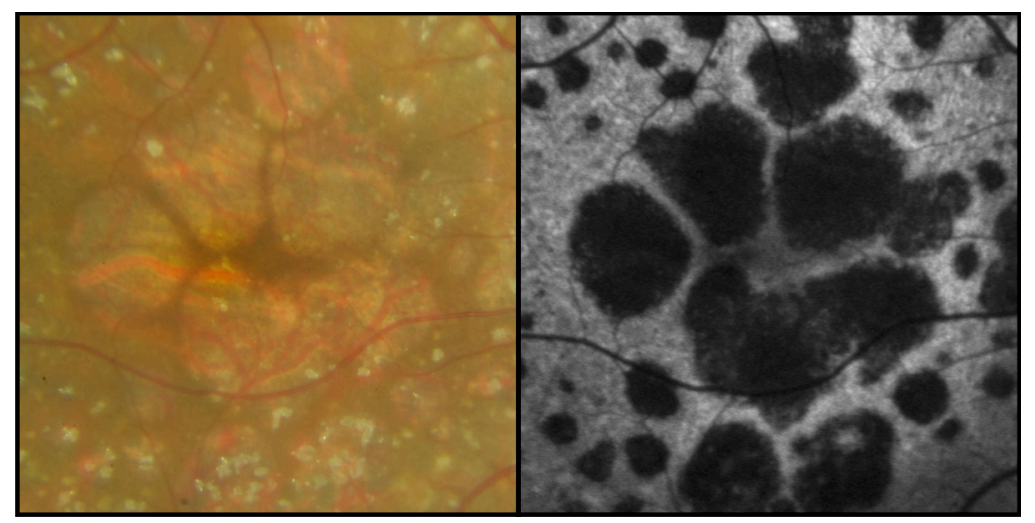

CEO_13837_CE0-20-06-0943 figure 1.tiff

This article is protected by copyright. All rights reserved. 


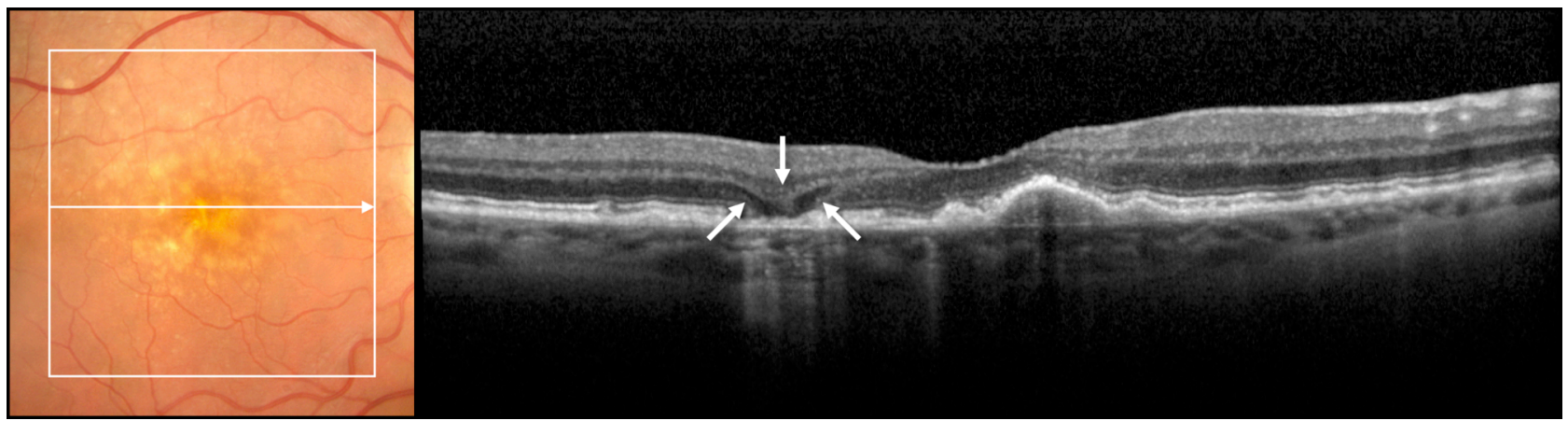

CEO_13837_CEO-20-06-0943 figure 2.tiff

This article is protected by copyright. All rights reserved. 


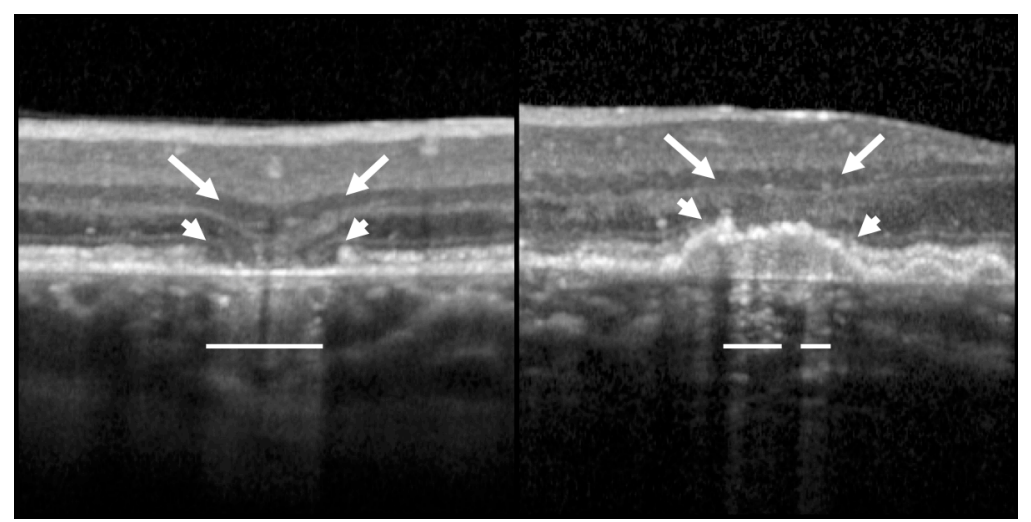

CEO_13837_CEO-20-06-0943 figure 3.tiff

This article is protected by copyright. All rights reserved. 


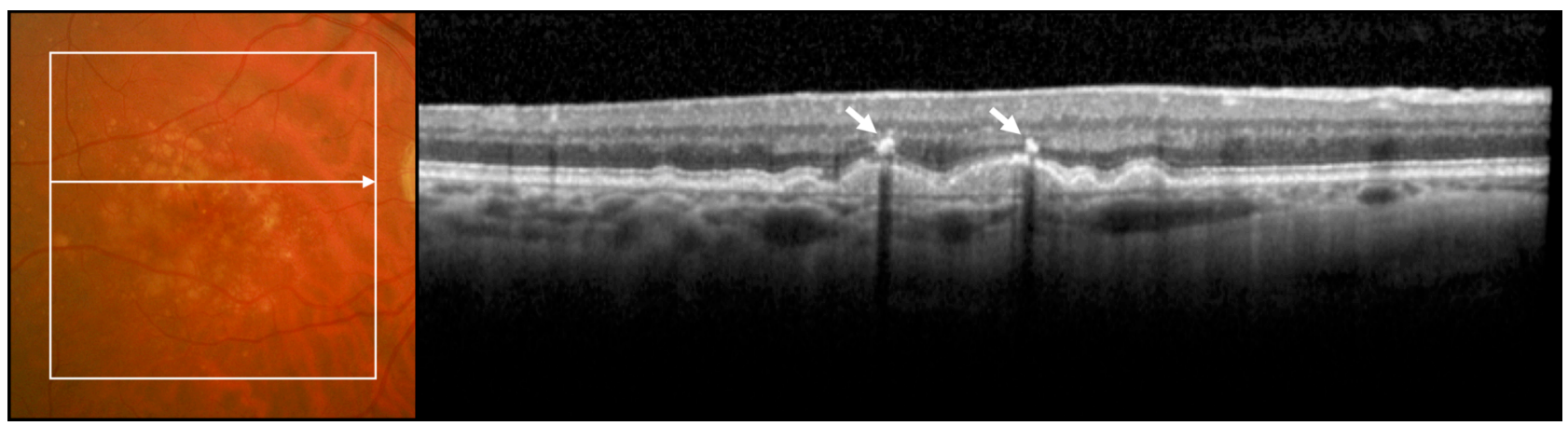

CEO_13837_CEO-20-06-0943 figure 4.tiff

This article is protected by copyright. All rights reserved. 


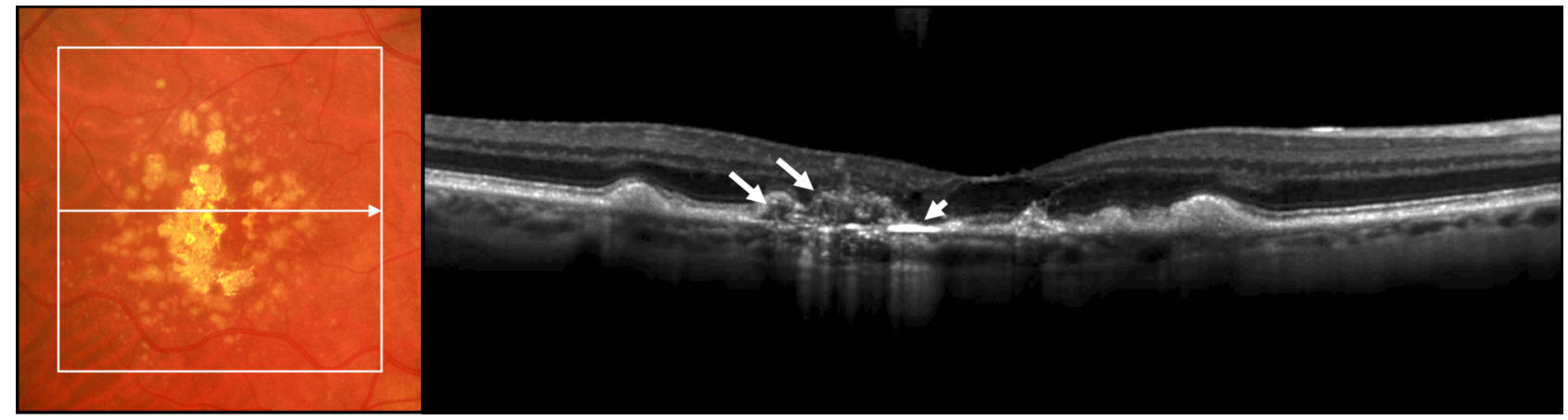

CEO_13837_CEO-20-06-0943 figure 5.tiff

This article is protected by copyright. All rights reserved. 


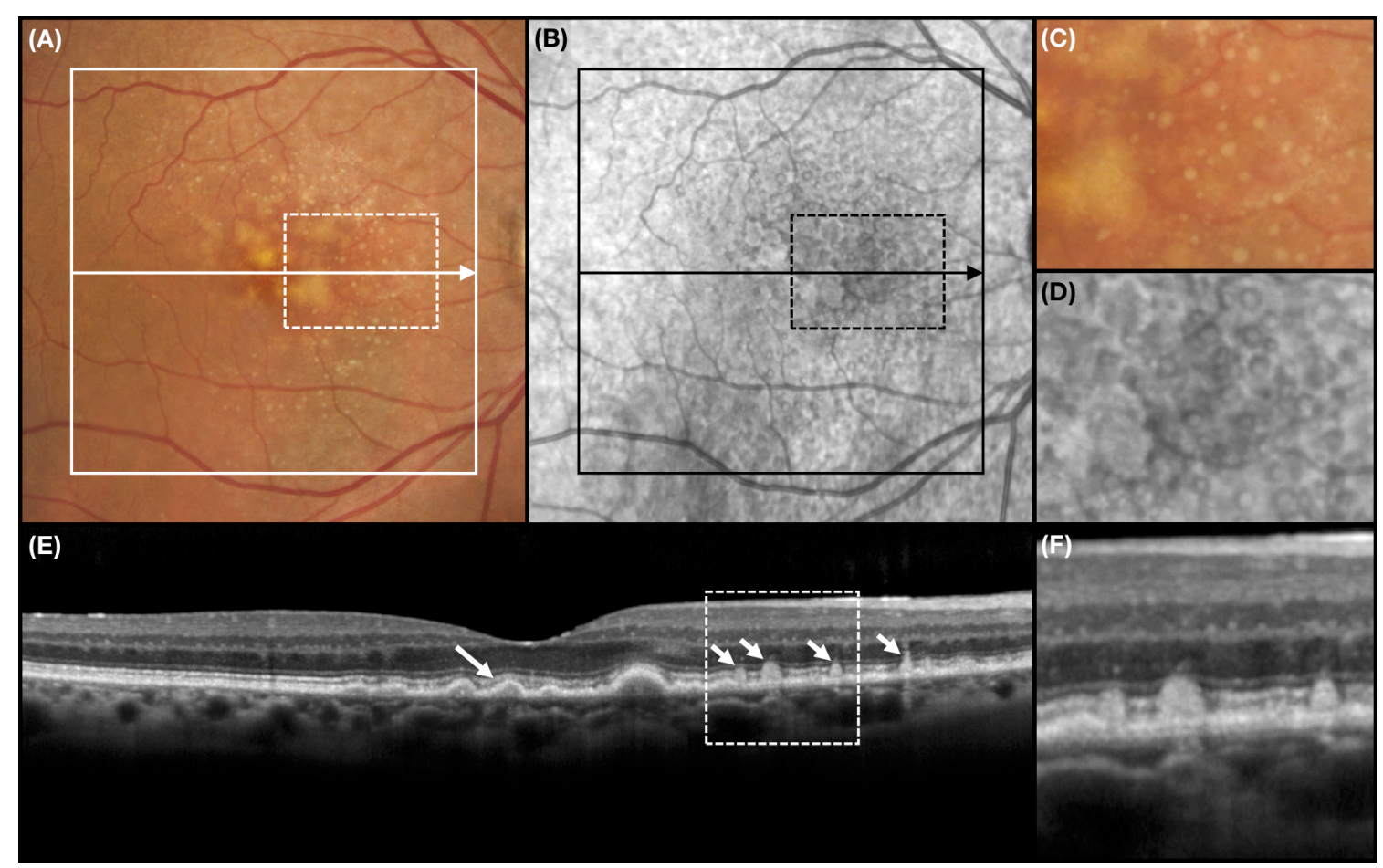

CE0_13837_CE0-20-06-0943 figure 6.tiff

This article is protected by copyright. All rights reserved. 


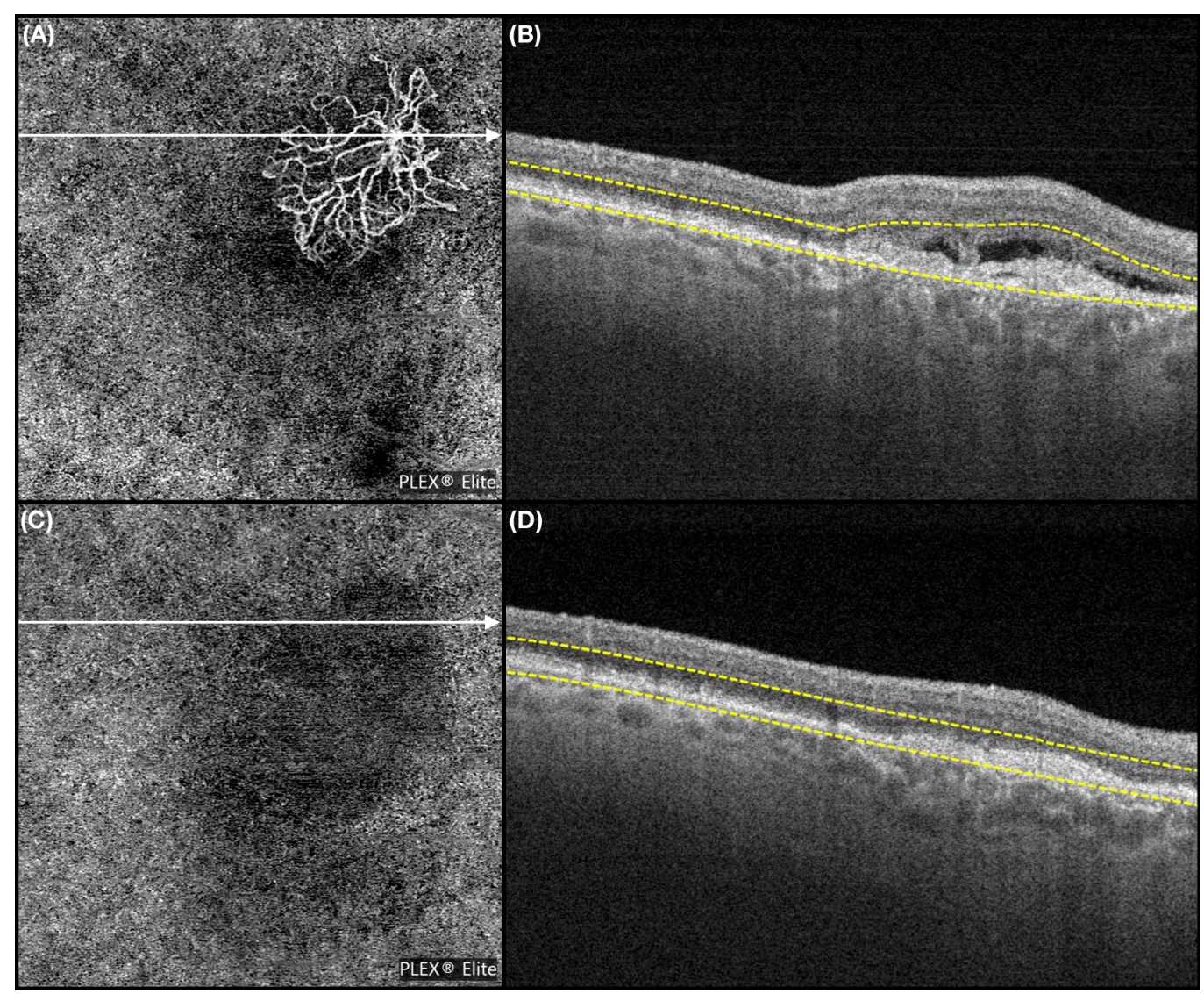

CEO_13837_CEO-20-06-0943 figure 7.tiff

This article is protected by copyright. All rights reserved. 

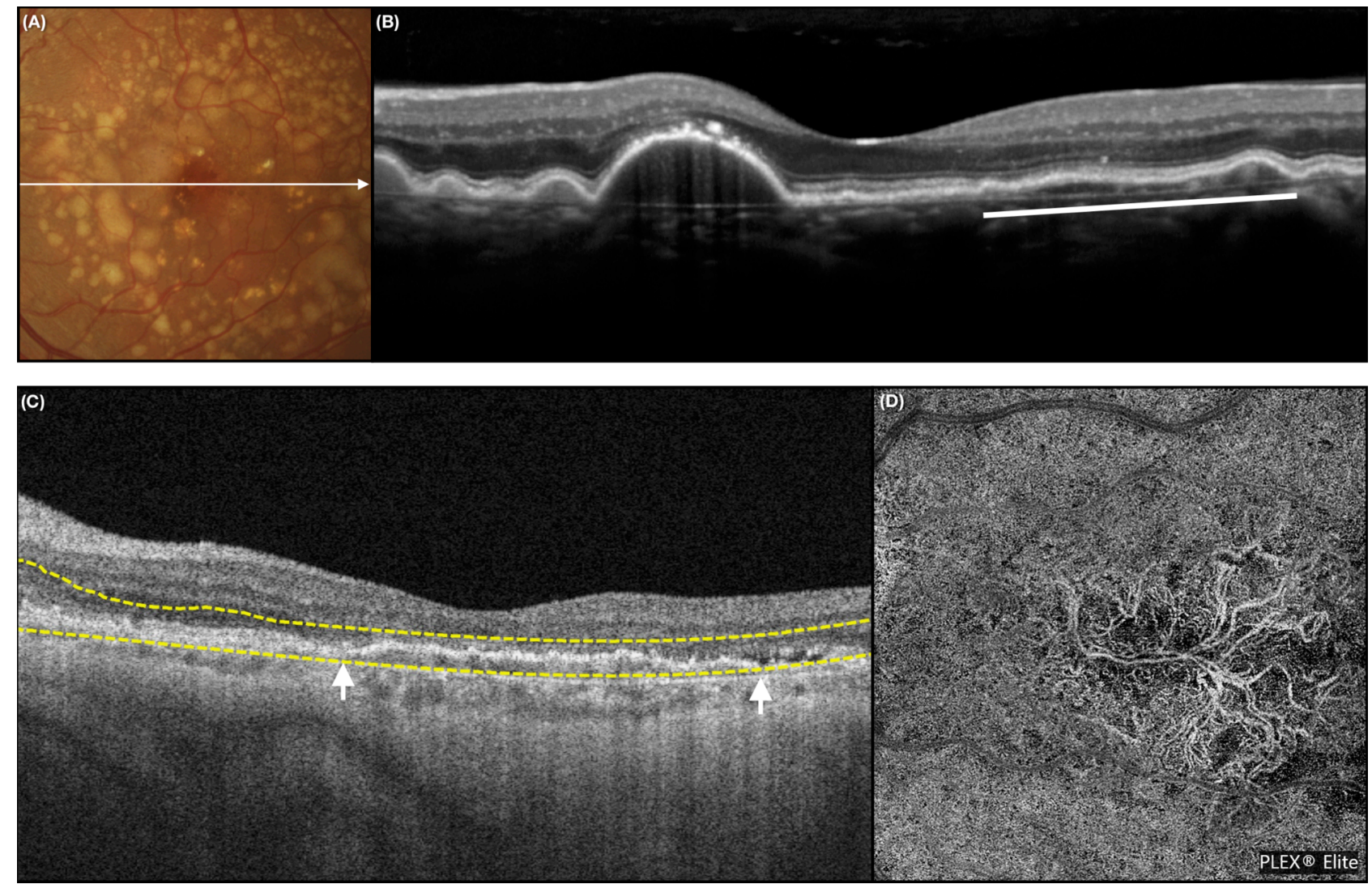

CEO_13837_CEO-20-06-0943 figure 8.tiff

This article is protected by copyright. All rights reserved. 


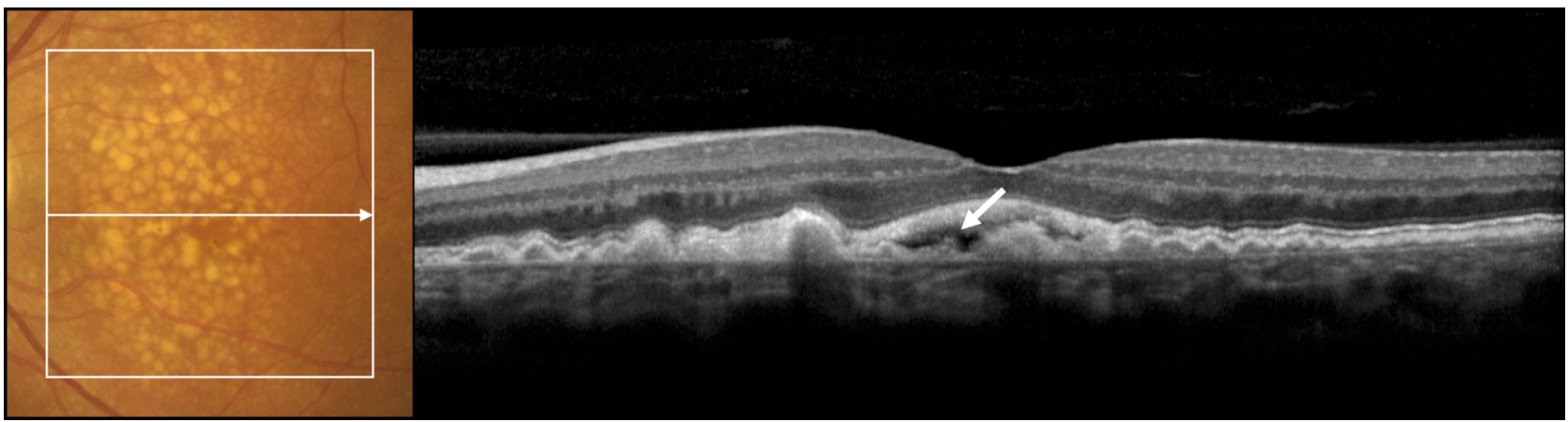

CEO_13837_CEO-20-06-0943 figure 9.tiff

This article is protected by copyright. All rights reserved. 